\title{
DESIGN, PRODUCE AND CONTROL OF A 2-DOF UPPER LIMB EXOSKELETAL ROBOT
}

\author{
A. Yavuz', E. Akdoğann,", M. E. Aktan³, A. T. Koru²
}

\begin{abstract}
Exoskeletal robots are used as high-tech products in the military, health and industrial applications. The integration of robots and humans offers new opportunities for the creation of new assistive technologies that can be used in biomedical, industrial and military applications. This paper presents the mechanical design, modeling and simulation of 2 degrees of freedom (DOF) upper limb exoskeletal robot. The system can be used both for supporting load lifting and for rehabilitation of upper limbs. A load cell was used to measure the applied load. Encoders were used to measure the shoulder and elbow joint angles. An electromyograph was developed to measure muscular activation. In this study, simulation was conducted with the PID position control, however the system hardware is applicable for a force control architecture.
\end{abstract}

\section{Keywords: Exoskeletal robot, EMG, Biomechatronics}

\section{INTRODUCTION}

In the growing research area of wearable robotics, exoskeletons are designed and developed to assist human limbs in various tasks. They are used in various areas such as military, medical and industrial applications in order to support the motion of human limbs. In the medical applications, the main purpose of the use of the exoskeleton robot is to support and rehabilitate the limbs.

The development of exoskeletons has begun in 1950s. There are various researches on exoskeletal robots in the literature. Some of these studies were developed for the load carrying applications, some were developed as the mobility assistants for elderly and disabled people, and some were developed for the rehabilitation purposes.

Heavy loads can typically be transported by devices such as cranes, wheeled vehicles and industrial robots. Despite the widespread use of these devices in industry, many tasks are still performed manually by workers. Manual handling activities are closely related to work-related musculoskeletal disorders [1]. Various exoskeletons are developed to help workers. Walsh et al. [2] designed an exoskeleton to transmit payload forces to the ground during the walking cycle. E. Yagi et al. [3] presented a system which helps agricultural laborer to carry heavy loads. Kim et al. [4] designed a lower limb exoskeleton to help the user to walk and to stand up from sitting in loaded conditions. Fontana et al. [5] designed 22-DOF electrically powered full-body exoskeleton to transport and to handle heavy loads up to $50 \mathrm{kgs}$, with one hand at worst-load conditions. Ghobj et al. [6] designed 4-DOF pneumatically actuated upper limb exoskeleton suit which is able to carry $8 \mathrm{kgs}$. Huysamen et al. [7] designed an exoskeleton to provide mechanical assistance to the body during lifting and lowering tasks to reduce body loading, back muscle activity and contact pressure. The system includes servo mechanisms and haptic devices. Exoskeletons are used to carry dangerous jobs as well as lifting loads in the industry. Umetani et al. [8] designed an exoskeleton system to be used in dangerous areas such as underwater and space etc. Yu et al. [9] designed a 7-DOF upper-limb exoskeleton for helping refractory construction operations in furnaces.

Various exoskeletons have been developed in order to improve the quality of lives of the old and disabled people and to help them in their daily life. Kiguchi et al. [10] designed an EMG controlled system to support the old and disabled people. Kong et al. [11] designed a wearable lower limb exoskeleton to help people for walking, sitting and standing up. Kiguchi et al. [12] designed a 3-DOF upper limb exoskeleton to assist physically disabled, injured, and elderly people. Besides these studies, there are many other examples of assistant exoskeletons [13$16]$.

Another application area of the exoskeletons is physical therapy and rehabilitation. Gupta and O'Malley [17] designed a 5 DOF haptic upper limb exoskeleton for training and rehabilitation in virtual environments. Vertechy et al. [18] proposed an exoskeleton design with a modular architecture for the rehabilitation of upper extremities. Ren et al. [19] designed an 8+2 DOF exoskeleton robot named IntelliArm. The robot is capable of controlling the upper limbs individually and simultaneously, and it helps to complete the rehabilitation. Martinez

\footnotetext{
This paper was recommended for publication in revised form by Tolga Taner

1 Tara Robotik Otomasyon ve Makine Imalat San. Tic. Ltd. Şti., Istanbul, Turkey

2 Department of Mechatronics Engineering, Yıldız Technical University, Istanbul, Turkey

${ }^{3}$ Department of Mechatronics Engineering, Bartın University, Bartın, Turkey

${ }^{\star} E$-mail address: eakdogan@yildiz.edu.tr

Orcid id: ${ }^{1} 0000-0002-2373-9284,{ }^{2} 0000-0003-1223-2725,{ }^{3} 0000-0001-7058-8362,{ }^{4}$ 0000-0001-8191-2324

Manuscript Received 27 February 2018, Accepted 25 May 2018
} 
et al. [20] designed a 3-DOF wrist gimbal for forearm and wrist rehabilitation. Yalçın [21] designed an exoskeleton named AssistOn-Arm for the upper limb rehabilitation. There are many other examples of exoskeletons for rehabilitation [22 - 26] apart from these studies.

In this paper, we present the design and production of a low-cost 2-DOF exoskeletal robot system for upper limbs. The main aim of this system is providing a low-cost solution with a novel exoskeleton mechanism design. The dynamic models and simulations are presented. The position based controller are used to control the system. In the system, there are position sensors for the measurement of the joint range of motions and force sensors to measure joint forces. Unlike other systems, the force sensor was placed in continuous contact with the palm of the hand in the mechanical design. Both biological and biomechanical parameters can be used as feedback. The system can be used to achieve therapeutic exercises, to measure the biomechanical parameters or to help workers in load carrying applications.

\section{FUNCTIONAL REQUIREMENTS AND DESIGN PARAMETERS}

Functional requirements and design parameters of developed system are listed in Table 1.

Table 1. Functional Requirements and Design Parameters

\begin{tabular}{|l|l|}
\hline \multicolumn{1}{|c|}{ Functional Requirements } & \multicolumn{1}{c|}{ Design Parameters } \\
\hline Has to be ergonomic & $\begin{array}{l}\text { The robot length is adjustable in sake of the comfort of } \\
\text { the user and to be used by various size of arms }\end{array}$ \\
\hline Proper movement ability & 2 DOF to perform shoulder and elbow movements \\
\hline The operator should carry $5 \mathrm{~kg}$ load easily & Motor torques and static calculations \\
\hline Has to be light and strong & 6013 T6 aluminum material \\
\hline
\end{tabular}

\section{MECHANICAL DESIGN}

The system has 2-DOF to perform shoulder and elbow movements. The design parameters are considered during the mechanical design process. Static analysis is performed with respect to load capacity of the system. The CAD drawings and photos of the system can be seen in Figure 1. The design is improved with respect to static analysis. The robot has an ergonomic design for which the length of the mechanism can be adjusted according to the arm length of the user. It is mobile thanks to the lockable wheels. The system is proper to use both arms. The handle includes a force sensor.

\section{STATIC ANALYSIS}

Free body diagram (FBD) of the robotic system can be seen in Figure 2. Static calculations are evaluated with respect to the FBD. Static equations for the $|\mathrm{CD}|$ part are:

$$
\begin{gathered}
\rightarrow+\sum F_{x}=0 \quad \Rightarrow \quad C_{x}=0 \\
+\uparrow \sum F_{y}=0 \quad \Rightarrow \quad C_{y}-W_{l o a d}-W_{l l}=0 \\
W_{\text {load }}(\max )=10 \cdot g \quad \text { and } \quad W_{l l}=m_{l l} \cdot g
\end{gathered}
$$

where $F_{x}$ denotes the force in $\mathrm{x}$ direction, $F_{y}$ the force in y direction, $C_{y}$ the force acting at $\mathrm{C}$ point, $W$ the weight, $m$ the mass and $g$ the gravity.

The static calculations are performed by isolating the links. First, the effects of load and the link weight on lower arm link $|\mathrm{CD}|$ are calculated. Then, the results are transformed to the upper limb, and static analysis is performed for the upper limb. According to the static analysis, the region A requires special consideration since it has the most bending. Analysis results are shown in Fig. 3 and 4.

As can be seen from the analysis, the yield strength for $6013 \mathrm{~T} 6$ aluminum material is $2.750 \times 10^{8}$. This value is not exceeded for both links. As a result, the link thicknesses are decreased. 
Journal of Thermal Engineering, Research Article, Vol. 5, No. 2, Special Issue 9, pp. 119-130, February, 2019

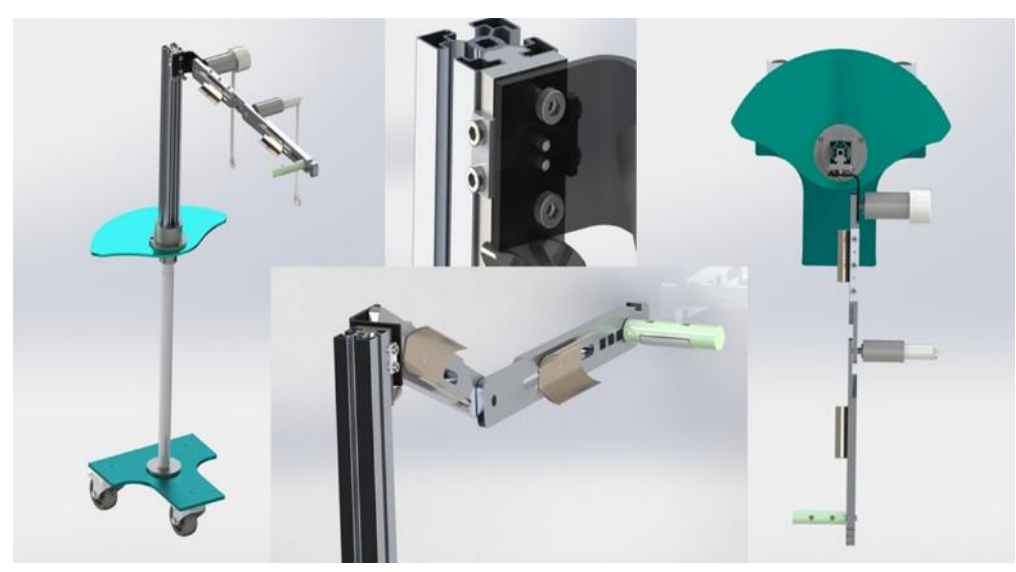

(a)
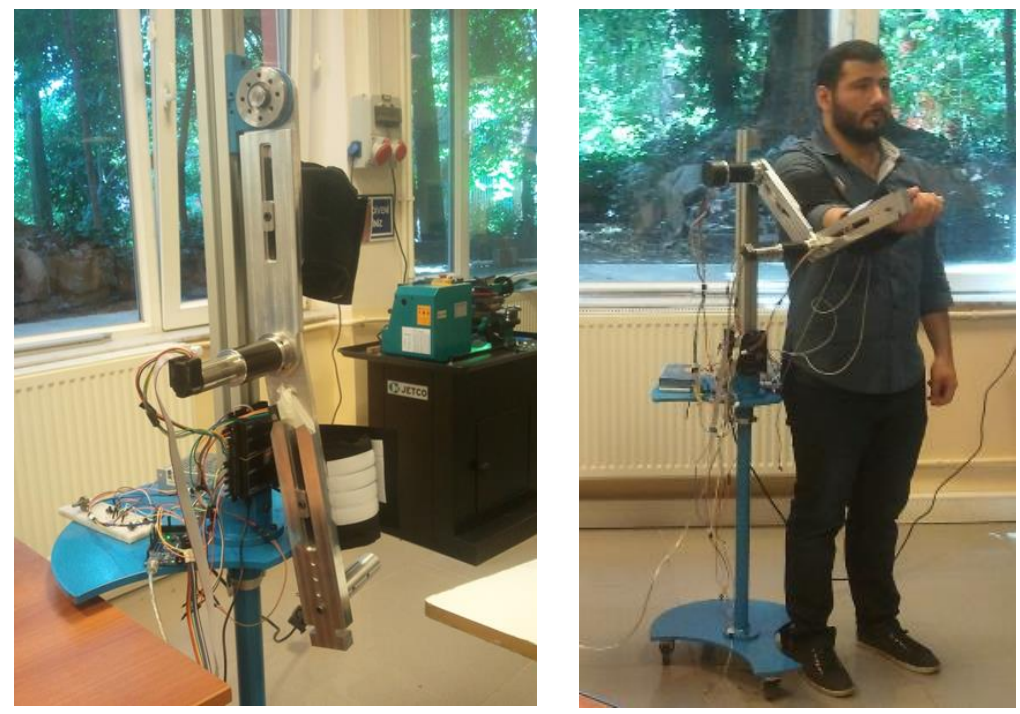

(b)

Figure 1. (a) CAD Drawings of robot, (b) final mechanism

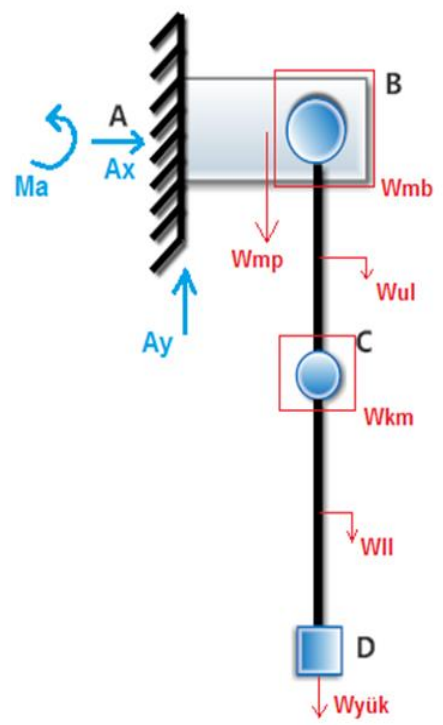

Figure 2. Free body diagram at $0^{\circ}$ 


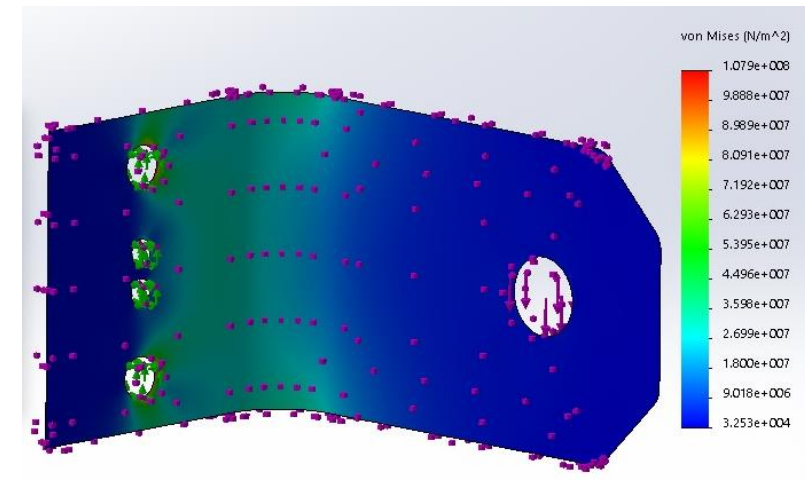

Figure 3. Static strain analysis of part A

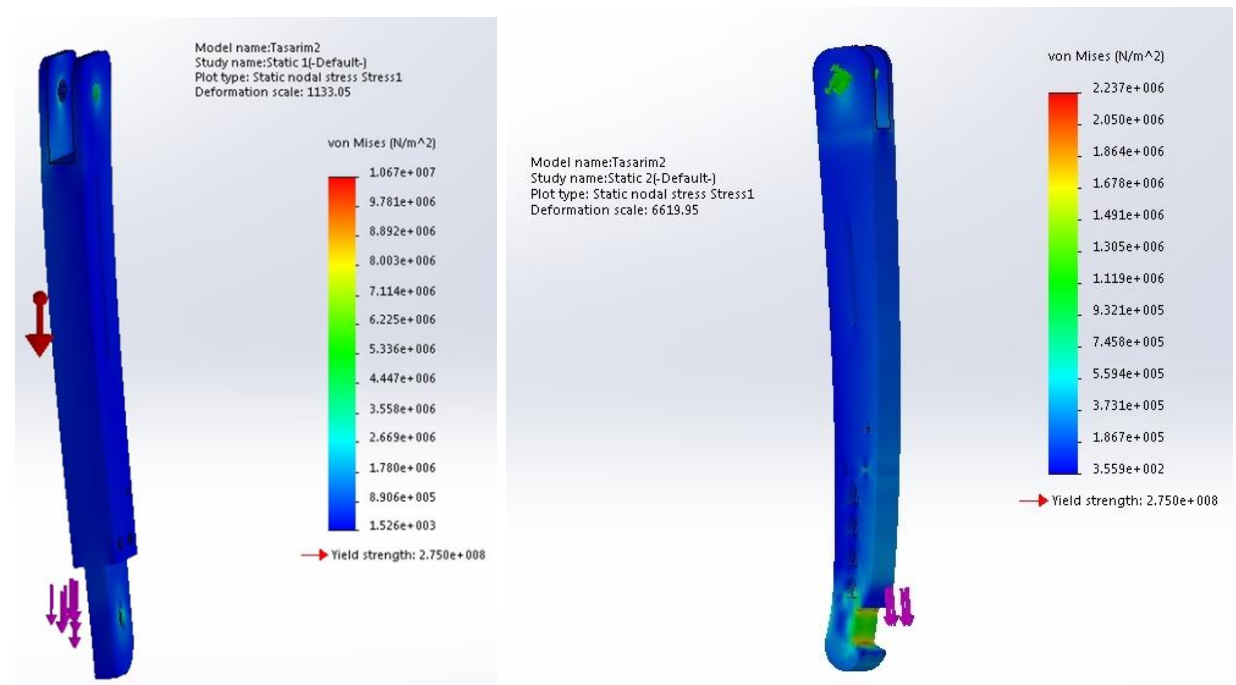

Figure 4. Upper and lower arm parts static analysis with a $5 \mathrm{~kg}$ load

The resulting links are bent off-axis. What occurred due to applied force is not distributed even over the link. In practice, there is a bearing in the inner surface which helps the load to be distributed evenly.

In order to choose motor and complete the system strength analysis, the static calculations for the 0 degree are not enough. The joint moments are changing with respect to limb angles. The moments are calculated by MATLAB. The results can be seen in Figure 5.

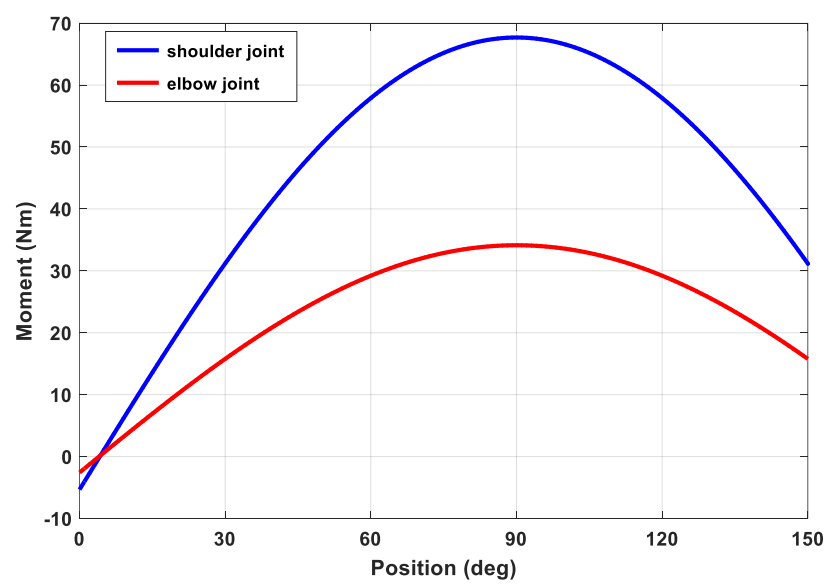

Figure 5. Angle-moment plot of shoulder and elbow link between $0-150$ degrees 


\section{KINEMATIC ANALYSIS}

Robot kinematics is used to analyze position, velocity and acceleration of the system [1]. Furthermore, the relation between end-effector position and joints are defined.

\section{Forward Kinematics}

The forward kinematics computes the position and the orientation of the robot end effector with respect to its base. The calculations are crucial for the control application. Forward kinematics is calculated using Denavit - Hartenberg convention. The links axes can be seen in Figure 6.

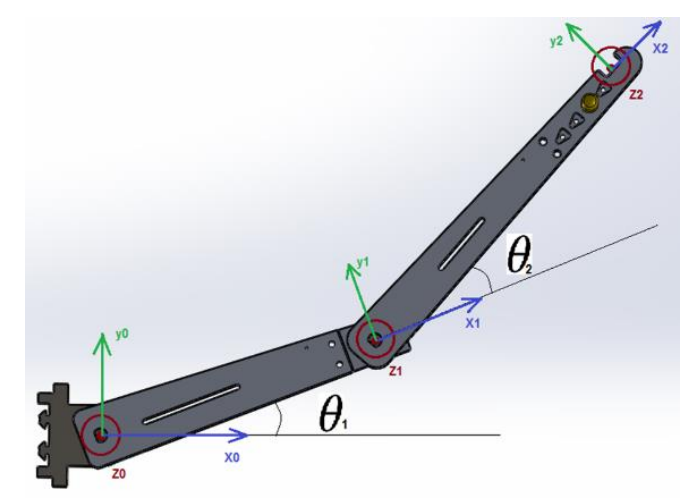

Figure 6. Coordinate systems for the robotic system

D-H parameters are given in Table 2.

Table 2. D-H Parameters

\begin{tabular}{|c|c|c|c|c|}
\hline \multirow{2}{*}{ Axis Number } & \multicolumn{4}{|c|}{ D-H Parameters } \\
\hline $\boldsymbol{i}$ & $\boldsymbol{\alpha}_{\boldsymbol{i}-\boldsymbol{1}}$ & $\boldsymbol{a}_{\boldsymbol{i}-\boldsymbol{1}}$ & $\boldsymbol{d}_{\boldsymbol{i}-\boldsymbol{1}}$ & $\boldsymbol{\theta}_{\boldsymbol{i}-\boldsymbol{1}}$ \\
\hline 1 & 0 & 0 & 0 & $\theta_{1}$ \\
\hline 2 & 0 & $L_{1}$ & 0 & $\theta_{2}$ \\
\hline 3 & 0 & $L_{2}$ & 0 & 0 \\
\hline
\end{tabular}

According to the D-H table, transformations are,

$$
T_{0}^{3}=T_{0}^{1} \cdot T_{1}^{2} \cdot T_{2}^{3}
$$

$T_{0}{ }^{3}$ is a $4 \times 4$ homogeny transformation matrix representing the end-effector position and orientation.

$$
\begin{gathered}
T_{0}^{1}=\left[\begin{array}{cccc}
c \theta_{1} & -s \theta_{1} & 0 & 0 \\
s \theta_{1} & c \theta_{1} & 0 & 0 \\
0 & 0 & 1 & 0 \\
0 & 0 & 0 & 1
\end{array}\right] \quad T_{1}^{2}=\left[\begin{array}{cccc}
c \theta_{2} & -s \theta_{2} & L_{1} & 0 \\
s \theta_{2} & c \theta_{2} & 0 & 0 \\
0 & 0 & 1 & 0 \\
0 & 0 & 0 & 1
\end{array}\right] \quad T_{2}^{3}=\left[\begin{array}{cccc}
1 & 0 & 0 & L_{2} \\
0 & 1 & 0 & 0 \\
0 & 0 & 1 & 0 \\
0 & 0 & 0 & 1
\end{array}\right] \\
T_{0}^{3}=\left[\begin{array}{cccc}
c\left(\theta_{1}+\theta_{2}\right) & -s\left(\theta_{1}+\theta_{2}\right) & 0 & L_{2} \cdot\left[c\left(\theta_{1}+\theta_{2}\right)\right]+L_{1} \cdot c \theta_{1} \\
s\left(\theta_{1}+\theta_{2}\right) & -c\left(\theta_{1}+\theta_{2}\right) & 0 & L_{2} \cdot\left[s\left(\theta_{1}+\theta_{2}\right)\right]+L_{1} \cdot s \theta_{1} \\
0 & 0 & 1 & 0 \\
0 & 0 & 0 & 1
\end{array}\right]
\end{gathered}
$$

\section{Inverse Kinematics}

The inverse kinematics calculates the joint angles for the given end-effector position and orientation. For the serial manipulators, the inverse kinematics is more complex then forward kinematics due to nonlinear equations. Inverse kinematics is given as follows: 
Journal of Thermal Engineering, Research Article, Vol. 5, No. 2, Special Issue 9, pp. 119-130, February, 2019

$$
\begin{gathered}
{\left[T_{0}^{1}\right]^{-1} \cdot T_{0}^{3}=T_{1}^{2} \cdot T_{2}^{3}} \\
{\left[T_{0}^{1}\right]^{-1}=\left[\begin{array}{cccc}
c \theta_{1} & s \theta_{1} & 0 & 0 \\
-s \theta_{1} & c \theta_{1} & 0 & 0 \\
0 & 0 & 1 & 0 \\
0 & 0 & 0 & 1
\end{array}\right] \quad T_{0}^{3}=\left[\begin{array}{cccc}
c\left(\theta_{1}+\theta_{2}\right) & -s\left(\theta_{1}+\theta_{2}\right) & 0 & p_{x} \\
s\left(\theta_{1}+\theta_{2}\right) & -c\left(\theta_{1}+\theta_{2}\right) & 0 & p_{y} \\
0 & 0 & 1 & p_{z} \\
0 & 0 & 0 & 1
\end{array}\right]} \\
T_{1}^{2}=\left[\begin{array}{cccc}
c \theta_{2} & -s \theta_{2} & L_{1} & 0 \\
s \theta_{2} & c \theta_{2} & 0 & 0 \\
0 & 0 & 1 & 0 \\
0 & 0 & 0 & 1
\end{array}\right] \quad T_{2}^{3}=\left[\begin{array}{cccc}
1 & 0 & 0 & L_{2} \\
0 & 1 & 0 & 0 \\
0 & 0 & 1 & 0 \\
0 & 0 & 0 & 1
\end{array}\right]
\end{gathered}
$$

The 12 equations are derived after the matrix multiplications. Among 12 equations, the following 2 equations are used to solve inverse kinematics problem.

$$
\begin{aligned}
& c \theta_{1} \cdot p_{x}+s \theta_{1} p_{y}=L_{1}+L_{2} \cdot c \theta_{2} \\
& -s \theta_{1} \cdot p_{x}+c \theta_{1} \cdot p_{y}=L_{2} \cdot s \theta_{2}
\end{aligned}
$$

After squaring the both sides and several algebraic manipulations, the following equations yield

$$
\begin{gathered}
p_{x}^{2}+p_{y}^{2}=L_{1}^{2}+2 L_{1} L_{2} c \theta_{2}+L_{2}^{2} \\
c \theta_{2}=\frac{p_{x}^{2}+p_{y}^{2}-L_{1}^{2}-L_{2}^{2}}{2 L_{1} L_{2}} \\
\theta_{2}=\operatorname{Atan2}\left( \pm \sqrt{1-\left[\frac{p_{x}^{2}+p_{y}^{2}-L_{1}^{2}-L_{2}^{2}}{2 L_{1} L_{2}}\right]^{2}}, \frac{p_{x}^{2}+p_{y}^{2}-L_{1}^{2}-L_{2}^{2}}{2 L_{1} L_{2}}\right) \\
\theta_{1}=\operatorname{Atan2}\left(p_{x}, p_{y}\right) \pm \operatorname{Atan} 2\left(\sqrt{p_{x}^{2}+p_{y}^{2}-\left(L_{1}+L_{2} * c \theta_{2}\right)^{2}}, L_{1}+L_{2} * c \theta_{2}\right)
\end{gathered}
$$

where $\theta_{1}$ and $\theta_{2}$ are the joint angles, $L_{1}$ and $L_{2}$ are the link lengths, $p_{\mathrm{x}}$ and $p_{\mathrm{y}}$ are the $\mathrm{x}$ and $\mathrm{y}$ positions of the end effector.

\section{ELECTRONICS HARDWARE}

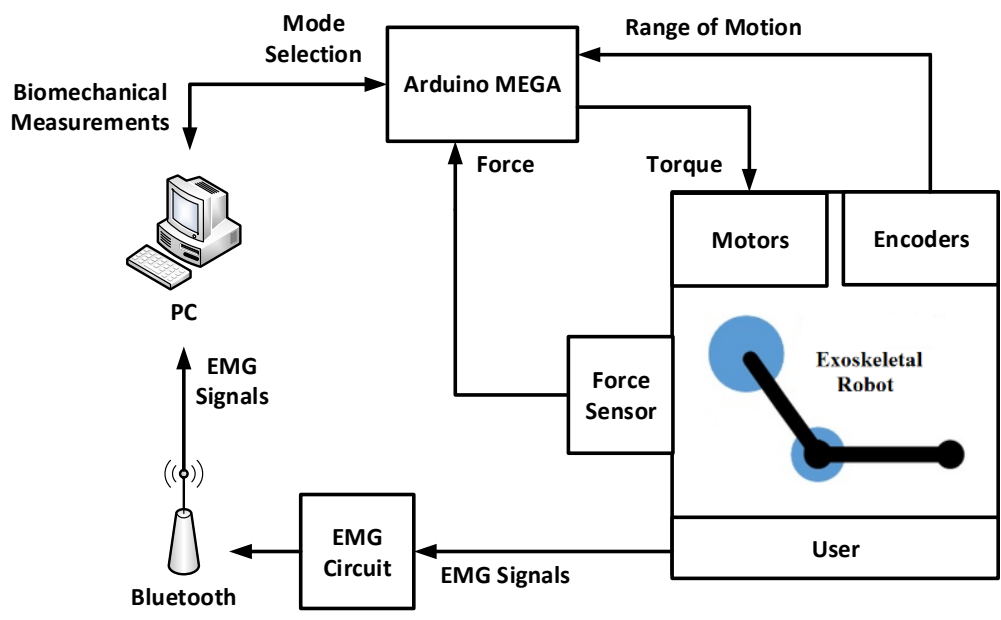

Figure 7. The electronics hardware block diagram 

2019

The electronic hardware block diagram is presented in Figure 7. The graphical user interface helps to choose mode and adjust mode parameters which are sent to the Arduino MEGA microcontroller. These parameters are used in the control algorithms. All of the sensor outputs, such as force and position feedbacks, are collected in the Arduino MEGA. The EMG signals during the exercise are transferred to the PC via a Bluetooth module.

\section{Arduino MEGA}

Arduino MEGA microcontroller is used to collect the data from sensors, process the signals and implement control algorithm.

\section{Motor - Gear - Encoder}

Motors are chosen according to the load capacity and movement ability of the human arms. Motor torque should carry the load and weight of the arm. On the other hand, motor velocity and acceleration should be enough to perform elbow and shoulder movements. The motor - gear - encoder specifications are presented in Table 3 .

Table 3. Motor - gear - encoder specifications

\begin{tabular}{|c|c|c|}
\hline & Elbow Joint & Shoulder Joint \\
\hline Gear & Planetary Gearhead GP 42 C, 3-15 Nm & Planetary Gearhead GP 52 C, 4-30 Nm \\
\hline Motor & Maxon EC-max 30, 60 W & Maxon EC 60 flat, 100 W \\
\hline Encoder & Encoder HEDL 5540, 500 CPT & Encoder MILE, 1024 CPT \\
\hline Driver & ESCON 50/5 4-Q Servocontroller & ESCON 50/5 4-Q Servocontroller \\
\hline
\end{tabular}

\section{Electromyography (EMG) Device}

EMG signals are the electrical signals generated during muscular contraction. The signal processing steps to make EMG signals useful are presented in Figure 8.

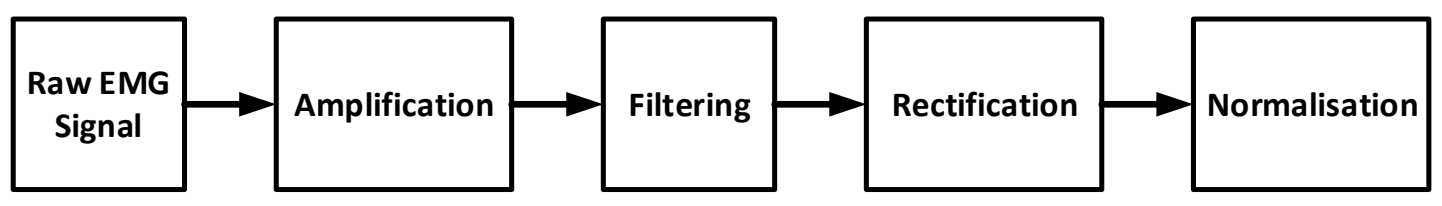

Figure 8. EMG signal processing

All of the steps can be realized by OP-AMP circuits. For more details, see [27].

\section{Force Sensor (FSR 402)}

The applied force at the end-effector is measured with FSR 402 Short Force Sensor. With the help of the force sensor and control algorithm, the operator never experiences a load more than $1 \mathrm{~kg}$. During the exercise mode, the sensor is used to measure patient force. Force sensor is placed at the end effector and can be seen in Figure 9. The sensor is in contact with hand palm continuously. This type of structure is not used in other similar systems.

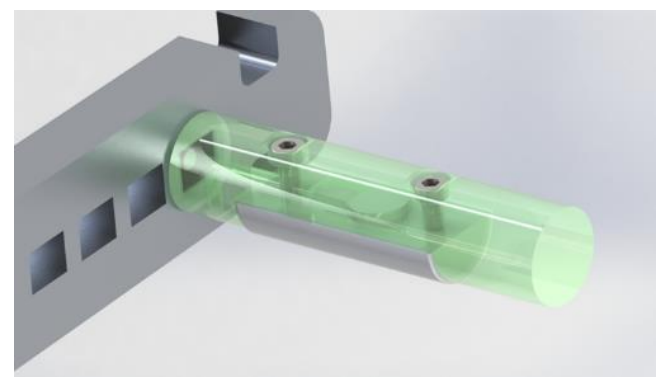

Figure 9. Force sensor is placed at the end effector handle. 


\section{Bluetooth Module (HC06)}

To communication between PC and robot is realized by HC06 Bluetooth module. The module communicates at $2.4 \mathrm{GHz}$ and has a range of 10 meters.

\section{MODELLING, CONTROL AND SIMULATION}

\section{Mathematical Modelling}

In this section, the mathematical models are presented. For the elbow motion, only the lower arm is active whereas both the lower and upper arms are active for the shoulder motion. DC motor model can be seen in Figure 10 .

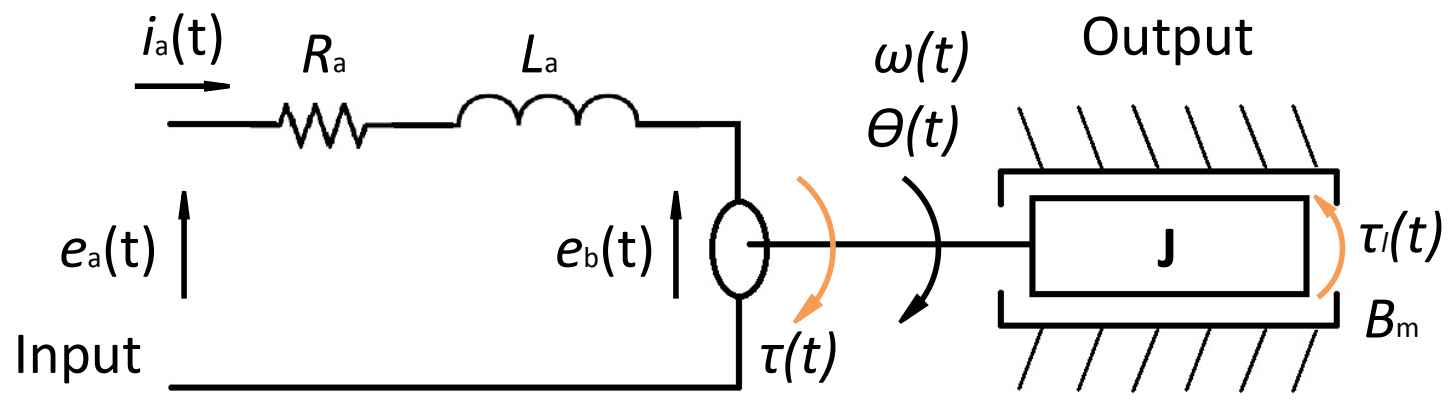

Figure 10. DC motor model

In the corresponding figure, $e_{a}(t)$ is the armature voltage, $i_{a}(t)$ armature current, $R_{a}$ resistor, $L_{a}$ inductor, $e_{b}(t)$ back EMF, $\omega(t)$ angular velocity, $\theta(t)$ angular position, $b_{m}$ viscous friction, $\tau$ torque, $K_{b}$ is the back EMF constant,

According to Kirchoff current law:

$$
e_{\mathrm{a}}=i_{a} \cdot R_{a}+\frac{d i_{a}}{d t} \cdot L_{a}+e_{b}
$$

Furthermore,

$$
\begin{aligned}
& \tau_{m}=i_{a} \cdot K_{t} \\
& e_{b}=K_{b} \cdot \omega_{m}
\end{aligned}
$$

After combining (14) with (15) and (16), the equations (17) and (18) yields.

$$
\begin{gathered}
e_{a}=\frac{\tau_{m}}{K_{t}} \cdot R_{a}+\frac{\tau_{m}}{K_{t}} \cdot s \cdot L_{a}+K_{b} \cdot s \cdot \theta_{m} \\
\tau_{m}=K_{t} \frac{\left(e_{a}-K_{b} \cdot \theta_{m} \cdot s\right)}{R_{a}+L_{a} \cdot s}
\end{gathered}
$$

\section{Elbow Movement}

$$
\begin{gathered}
\frac{N_{m 1}}{N_{11}}=\eta_{1} \Rightarrow \theta_{m 1}=\theta_{1} \frac{N_{11}}{N_{m 1}}=\theta_{1} \frac{1}{\eta_{1}} \\
J_{e q 1}=J_{m 1}+J_{1}\left(\frac{1}{\eta_{1}}\right)^{2} \quad \text { and } \quad b_{e q 1}=b_{m}+b_{g}+b_{r}\left(\frac{1}{\eta_{1}}\right)^{2} \\
\frac{\theta_{m 1}}{\tau_{m 1}}=\frac{1}{J_{e q 1} s^{2}+b_{e q 1} \cdot s}
\end{gathered}
$$

where $\eta_{1}$ is the gear ratio, $J_{i}$ is the moment of inertia, $b_{r}$ and $b_{g}$ are rotor and gear frictions, $N_{i}$ are the number of teeth of the gears. If $\tau_{m 1}$ is replaced; 
Journal of Thermal Engineering, Research Article, Vol. 5, No. 2, Special Issue 9, pp. 119-130, February, 2019

$$
\frac{\theta_{m 1}}{K_{t 1} \frac{\left(e_{a 1}-K_{b 1} \theta_{m 1} \cdot s\right)}{R_{a 1}+L_{a 1} \cdot s}}=\frac{1}{J_{e q 1} s^{2}+b_{e q 1} \cdot s}
$$

After some algebraic manipulations, following transfer functions are derived:

$$
\begin{gathered}
\frac{\theta_{m 1}}{e_{a 1}}=\frac{K_{t 1}}{J_{e q 1} L_{a 1} s^{3}+\left(J_{e q 1} R_{a 1}+b_{e q 1} L_{a 1}\right) s^{2}+\left(b_{e q 1} R_{a 1}+K_{t 1} K_{b 1}\right) s} \\
\frac{\theta_{1}}{e_{a 1}}=\frac{K_{t 1} \cdot \eta_{1}}{J_{e q 1} L_{a 1} s^{3}+\left(J_{e q 1} R_{a 1}+b_{e q 1} L_{a 1}\right) s^{2}+\left(b_{e q 1} R_{a 1}+K_{t 1} K_{b 1}\right) s} \\
\frac{\theta_{1}}{e_{a 1}}=\frac{2.34}{2.741 \cdot 10^{-10} s^{3}+1.551 \cdot 10^{-6} s^{2}+0.00232 \cdot s}
\end{gathered}
$$

\section{Shoulder Movement}

$$
\begin{gathered}
\frac{N_{m 2}}{N_{12}}=\eta_{2} \Rightarrow \theta_{m 2}=\theta_{2} \frac{N_{12}}{N_{m 2}}=\theta_{2} \frac{1}{\eta_{2}} \\
J_{e q 2}=J_{m 2}+\left(J_{1}+J_{2}\right) \cdot\left(\frac{1}{\eta_{2}}\right)^{2} \quad \text { and } \quad b_{e q 2}=b_{m}+b_{g}+b_{r} \cdot\left(\frac{1}{\eta_{2}}\right)^{2} \\
\frac{\theta_{m 2}}{T_{m 2}}=\frac{1}{J_{e q 2} s^{2}+b_{e q 2} \cdot s}
\end{gathered}
$$

If $T_{\mathrm{m} 2}$ is replaced:

$$
\frac{\theta_{m 2}}{K_{t 2} \frac{\left(e_{a 2}-K_{b 2} \theta_{m 2} \cdot s\right)}{R_{a 2}+L_{a 2} \cdot s}}=\frac{1}{J_{e q 2} s^{2}+b_{e q 2} \cdot s}
$$

After some algebraic manipulations, following transfer functions are derived:

$$
\begin{gathered}
\frac{\theta_{m 2}}{e_{a 2}}=\frac{K_{t 2}}{J_{e q 2} L_{a 2} s^{3}+\left(J_{e q 2} R_{a 2}+b_{e q 2} L_{a 2}\right) s^{2}+\left(b_{e q 2} R_{a 2}+K_{t 2} K_{b 2}\right) s} \\
\frac{\theta_{2}}{e_{a 2}}=\frac{K_{t 2} \cdot \eta_{2}}{J_{e q 2} L_{a 2} s^{3}+\left(J_{e q 2} R_{a 2}+b_{e q 2} L_{a 2}\right) s^{2}+\left(b_{e q 2} R_{a 2}+K_{t 2} K_{b 2}\right) s} \\
\frac{\theta_{2}}{e_{a 2}}=\frac{4.571}{1.27 \cdot 10^{-9} s^{3}+6.052 \cdot 10^{-6} s^{2}+0.007486 s}
\end{gathered}
$$

\section{Simulation and Control}

The simulations are used to tune the PID coefficients. The impedance control method will be implemented in future works. Simmechanics model can be seen in Figure 11. 


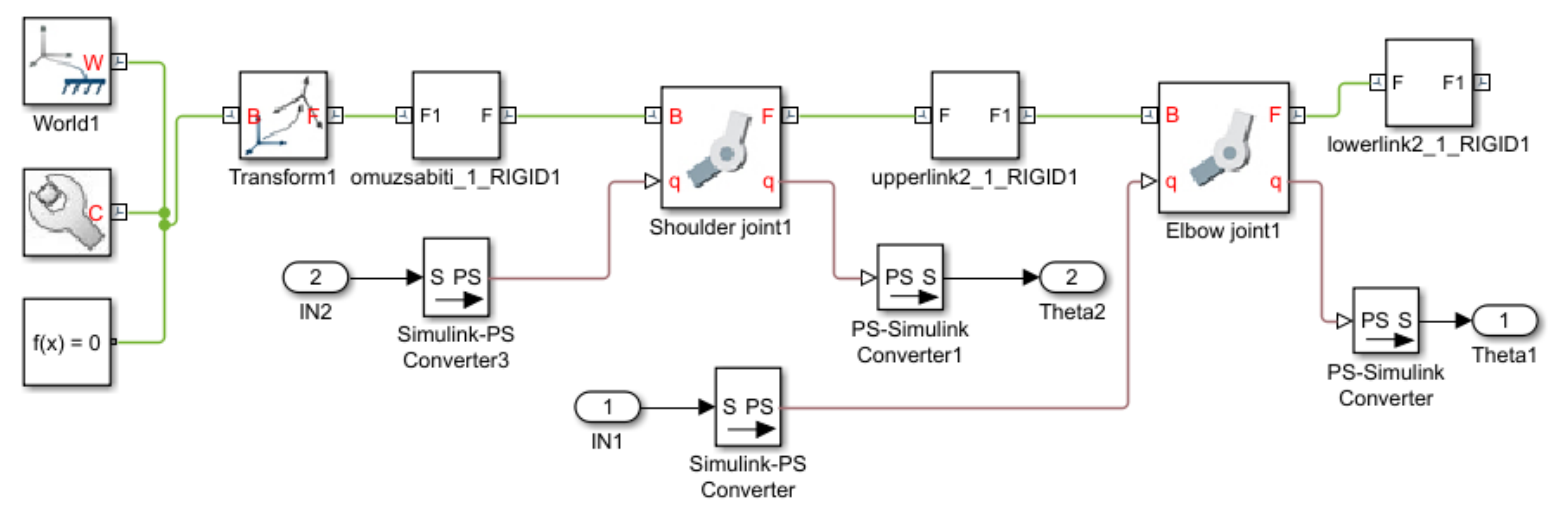

Figure 11. Simmechanics model of the exoskeletal robot

The PID coefficients are calculated using the "Tune Parameters Function" of the MATLAB for both elbow and shoulder joint. The simulations results after PID design can be seen in figures 12 and 13. Position error is less than 0.01 radians.
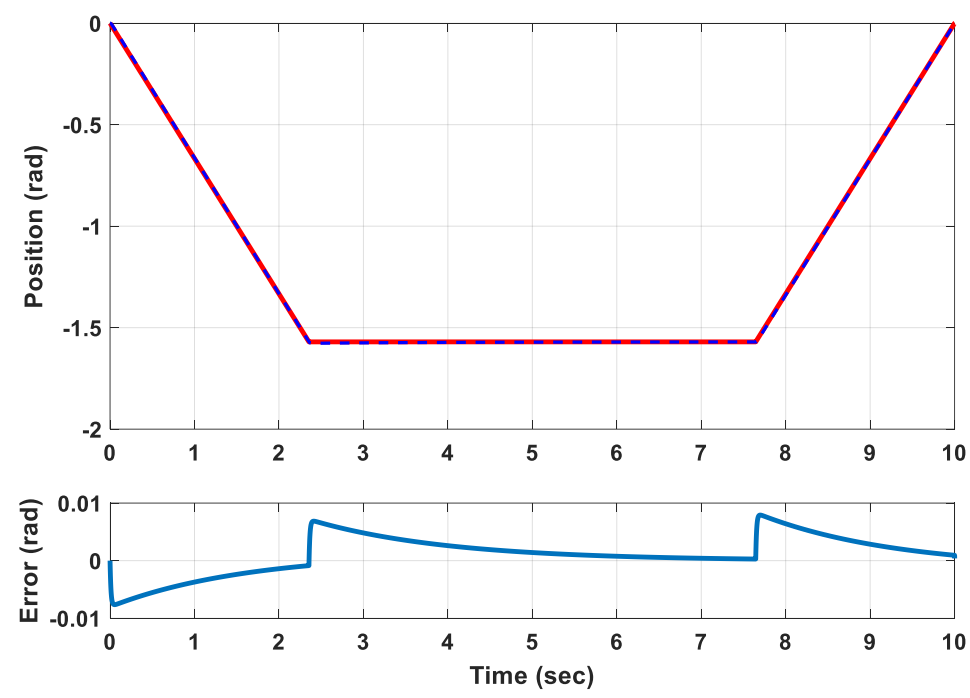

Figure 12. Elbow joint input and output signals and error
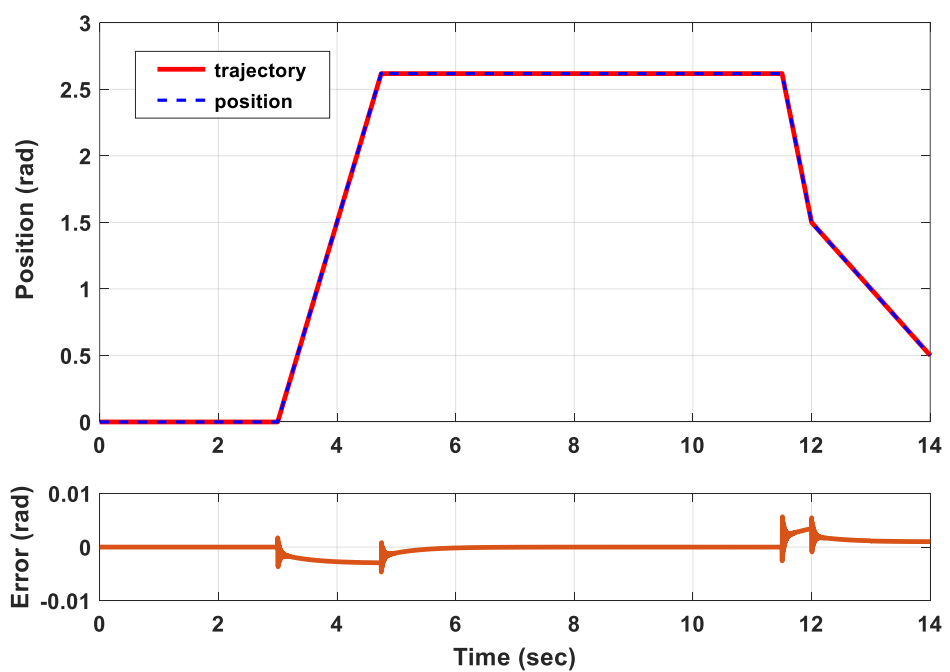

Figure 13: Shoulder joint input and output signals and error 


\section{OPERATION MODES OF THE EXOSKELETAL ROBOT}

The developed exoskeletal robot includes 3 different modes: (1) load lifting, (2) passive exercise and (3) resistive exercise modes.

\section{Load Lifting Mode}

This mode helps the operator to lift a load. While the operator is carrying a load, the EMG signals and the force sensors data are evaluated at the microcontroller. The control algorithms adjust the motor torque with respect to those data.

\section{Passive Exercise Mode}

The purpose of this mode is to rehabilitate the arm. The range of motion is entered from the GUI. The exoskeletal system performs the passive exercise in this range of motion. EMG signals are sent to the PC via Bluetooth module.

\section{Resistive Exercise Mode}

The patient works his/her muscles against the motor torques. The biomechanical parameters which are output of the force sensor, the joint angles and EMG signals are saved in a database.

\section{CONCLUSION}

In this study, a low cost 2-DOF exoskeletal robot is designed and controlled to perform therapeutic exercises and support workers in the load carrying tasks. The mechanical properties, electronics hardware, mathematical modelling and control algorithms are presented.

The developed exoskeletal robot can be used in the load lifting applications, performing therapeutic exercises and the measurement of biomechanical parameters. It can increase the life quality of old and disabled people. It can make the treatment at home possible.

In order to extend this study, the force-based controller structure can be developed. The system can be made lighter, more modular and wearable. Furthermore, the system will be tested with healthy subjects and patients.

\section{ACKNOWLEDGEMENTS}

The development of the robotic system is supported by TÜBİTAK 2241B under the project name "EMG Based Control of an Arm Exoskeletal System".

\section{NOMENCLATURE}

$\begin{array}{ll}F_{x} & \text { Force in x direction, } \mathrm{N} \\ F_{y} & \text { Force in y direction, } \mathrm{N} \\ C_{y} & \text { Force acting at C point, } \mathrm{N} \\ W & \text { Weight, } \mathrm{N} \\ m & \text { Mass, } \mathrm{kg} \\ g & \text { Acceleration due to gravity, } \mathrm{m} / \mathrm{s}^{2} \\ \theta_{1} & \text { Angle of first joint, degree } \\ \theta_{2} & \text { Angle of second joint, degree } \\ L_{1} & \text { Length of first link, } \mathrm{m} \\ L_{2} & \text { Length of second link, } \mathrm{m} \\ p_{x} & \text { Position of the end effector on the x axis, } \mathrm{m} \\ p_{y} & \text { Position of the end effector on the y axis, } \mathrm{m} \\ t & \text { Time, second } \\ e_{a} & \text { Armature voltage, } \mathrm{V} \\ i_{a} & \text { Armature current, } \mathrm{A} \\ R_{a} & \text { Resistor, } \Omega \\ L_{a} & \text { Inductor, } \mathrm{H} \\ e_{b} & \text { Back electromotive force, } \mathrm{V} \\ \omega(t) & \text { Angular velocity, rad } / \mathrm{s} \\ \theta(t) & \text { Angular position, rad } \\ b_{m} & \text { Viscous friction, } \mathrm{Nm} / \mathrm{s} \\ \tau & \text { Torque, Nm } \\ \eta & \text { Gear ratio } \\ J & \text { Inertia, kg m }{ }^{2} \\ N & \text { Number of teeth of gear }\end{array}$




\section{REFERENCES}

[1] J. Zurada. (2012). Classifying the risk of work related low back disorders due to manual material handling tasks. Expert Systems with Applications. 39(12), 11125-11134.

[2] C. J. Walsh, K. Pasch, H. Herr. (2006). An autonomous, underactuated exoskeleton for load-carrying augmentation. IEEE/RSJ International Conference on Intelligent Robots and Systems, 1410-1415.

[3] E. Yagi, D. Harada, M. Kobayashi. (2009). Upper-Limb Power-Assist Control for Agriculture Load Lifting. International Journal of Automation Technology, 3, 716-722.

[4] H. G. Kim, J. W. Lee, J. Jang, C. Han and S. Park (2013). Mechanical design of an exoskeleton for loadcarrying augmentation. IEEE ISR 2013, 1-5.

[5] M. Fontana, R. Vertechy, S. Marcheschi, F. Salsedo, M. Bergamasco. (2014). The Body Extender: A FullBody Exoskeleton for the Transport and Handling of Heavy Loads. IEEE Robotics \& Automation Magazine, 21(4), 34-44.

[6] S. Ghobj, A. Akl, A. El-Farr, M. Ayyash, J. Abu-Khalaf. (2017). Mechanical design for a cable driven upper limb exoskeleton prototype actuated by pneumatic rubber muscles. International Conference on Research and Education in Mechatronics (REM), 1-7.

[7] K.Huysamen, M.Looze, T. Bosch, J.Ortiz, S.Toxiri, L.W.O'Sullivan. (2018). Assessment of an active industrial exoskeleton to aid dynamic lifting and lowering manual handling tasks. Applied Ergonomics, 68, 125-131.

[8] Y. Umetani, Y. Yamada, T. Morizono, T. Yoshida, S. Aoki. (1999). "Skil Mate" wearable exoskeleton robot. IEEE International Conference on Systems, Man, and Cybernetics, 4, 984-988.

[9] H. Yu, I. S. Choi, K. L. Han, J. Y. Choi, G. Chung, J. Suh. (2018). Development of an upper-limb exoskeleton robot for refractory construction. Control Engineering Practice, 72, 104-113.

[10] K. Kiguchi, T. Tanaka, T. Fukuda. (2004). Neuro-fuzzy control of a robotic exoskeleton with EMG signals. IEEE Transactions on Fuzzy Systems, 12(4), 481-490.

[11] K. Kong, D. Jeon. (2006). Design and control of an exoskeleton for the elderly and patients. IEEE/ASME Transactions on Mechatronics, 11(4), 428-432.

[12] K. Kiguchi, M. H. Rahman, M. Sasaki, K. Teramoto. (2008). Development of a 3DOF mobile exoskeleton robot for human upper-limb motion assist. Robotics and Autonomous Systems, 56(8), 678-691.

[13] D. Naidu, R. Stopforth, G. Bright, S. Davrajh. (2011). A 7 DOF exoskeleton arm: Shoulder, elbow, wrist and hand mechanism for assistance to upper limb disabled individuals. AFRICON, 1-6.

[14] G. Ivanova, S. Bulavintsev, J. H. Ryu, J. Poduraev. (2011). Development of an Exoskeleton System for Elderly and Disabled People. International Conference on Information Science and Applications, 1-7.

[15] B. Chen, C. H. Zhong, X. Zhao, H. Ma, X. Guan, X. Li, F. Y. Liang, J. C. Y. Cheng, L. Qin, S. W. Law, W. H. Liao. (2017). A wearable exoskeleton suit for motion assistance to paralysed patients. Journal of Orthopaedic Translation, 11, 7-18.

[16] K. Huysamen, T. Bosch, M. Looze, K. S. Stadler, E. Graf, L. W. O'Sullivan. (2018). Evaluation of a passive exoskeleton for static upper limb activities. Applied Ergonomics, 70, 148-155.

[17] A. Gupta, M. K. O'Malley. (2006). Design of a haptic arm exoskeleton for training and rehabilitation. IEEE/ASME Transactions on Mechatronics, 11(3), 280-289.

[18] R. Vertechy, A. Frisoli, A. Dettori, M. Solazzi, M. Bergamasco. (2009). Development of a new exoskeleton for upper limb rehabilitation. IEEE International Conference on Rehabilitation Robotics, 188-193.

[19] Y. Ren, H. S. Park, L. Q. Zhang. (2009). Developing a whole-arm exoskeleton robot with hand opening and closing mechanism for upper limb stroke rehabilitation. IEEE International Conference on Rehabilitation Robotics, 761-765.

[20] J. A. Martinez, P. Ng, S. Lu, M. S. Campagna, O. Celik. (2013). Design of Wrist Gimbal: A forearm and wrist exoskeleton for stroke rehabilitation. IEEE 13th International Conference on Rehabilitation Robotics, 1-6.

[21] M. Yalçın. (2013). Design, implementation and control of a self-aligning full arm exoskeleton for physical rehabilitation. Master Thesis, Sabanc1 University.

[22] F. Zhang, L. Hua, Y. Fu, H. Chen, S. Wang. (2014). Design and development of a hand exoskeleton for rehabilitation of hand injuries. Mechanism and Machine Theory, 73, 103-116.

[23] J. Iqbal, H. Khan, N. G. Tsagarakis, D. G. Caldwell (2014). A novel exoskeleton robotic system for hand rehabilitation - Conceptualization to prototyping. Biocybernetics and Biomedical Engineering, 34(2), 79-89.

[24] Y. Ganesan, S. Gobee, V. Durairajah. (2015). Development of an Upper Limb Exoskeleton for Rehabilitation with Feedback from EMG and IMU Sensor. Procedia Computer Science, 76, 53-59.

[25] S. R. A. Jafri, M. B. A. Abbasi, S. M. U. A. Shah, A. Hanif, M. Usman. (2017). BIPATRON (Bionic parageliatron): A wearable robot for rehabilitation... Lets Walk!. 2017 First International Conference on Latest trends in Electrical Engineering and Computing Technologies (INTELLECT), 1-7.

[27] M. E. Aktan, İ. Göker, E. Akdoğan, B. Öztürk. (2017). Design, implementation and performance analysis of a microcontroller based wireless electromyography device. 2017 Medical Technologies National Congress, 1-4. 